\title{
The Bologna Open Cluster Chemical Evolution project: a large, homogeneous sample of Galactic open clusters
}

\author{
Angela Bragaglia \\ INAF-Osservatorio Astronomico di Bologna \\ via Ranzani 1, I-40127 Bologna, Italy \\ email: angela.bragaglia@oabo.inaf.it
}

\begin{abstract}
The Bologna Open Cluster Chemical Evolution (BOCCE) project is a photometric and spectroscopic survey of open clusters, to be used as tracers of the Galactic disk properties and evolution. The clusters parameters (age, distance, reddening, metallicity, and detailed abundances) are derived in a precise and homogeneous way. This will contribute to a solid, reliable description of the disk: the clusters parameters will be used, for instance, to determine the metallicity distribution in the Galactic disk and how it has evolved with time. We have concentrated on old open clusters and we have presently in our hands data for about 40 open clusters; we have fully analyzed the photometric data for about one half of them and the spectra for one quarter of them.
\end{abstract}

Keywords. techniques: photometric, techniques: spectroscopic, stars: abundances, HertzsprungRussell diagram, Galaxy: abundances, Galaxy: disk, open clusters and associations: general

\section{Introduction}

The distribution of metals with Galactocentric distance is a signature of how the disk formed (inside-out? with infalls, outflows, mergers, etc ?). To derive it we need to know, for a given population, metallicity, distance, and age. The metallicity distribution can be traced for instance by young stars and Cepheids, but they can only be used to describe the present-day situation. Planetary nebulae go further in the past; however, there is still no consensus on the radial gradient based on them, or on the time evolution, see e.g., Maciel et al. (2003) and Stanghellini et al. (2006) for opposite ideas.

Open clusters (OCs) are very useful tracers of the properties of the Galactic disk (e.g., Friel 1995) because i) they are seen over the entire disk, ii) their ages and distances are generally measurable with better precision than those of isolated field stars up to large distances, and iii) they cover the entire range of metallicities and ages of the disk. Studying OCs we may understand whether and how the metallicity distribution changed with time.

OCs have been used in the past to define the metallicity distribution, but also in this case results were not conclusive. Most researchers found a negative radial gradient (e.g., Friel et al. 2002, based on homogeneous, low resolution spectroscopy), but an alternative picture has been presented: Twarog, Ashman \& Anthony-Twarog (1997) invoked two flat distributions at about solar and sub-solar metallicity, with the discontinuity near a Galactocentric distance $\left(\mathrm{R}_{\mathrm{GC}}\right)$ of $10 \mathrm{kpc}$. More recently, the observations of OCs at large $\mathrm{R}_{\mathrm{GC}}$ 's, up to more than $20 \mathrm{kpc}$, seem to indicate a negative gradient in the inner region and a flattening in the outer disk, with the transition at 10-14 kpc: see Yong, Carney \& Teixera de Almeida (2005), Carraro et al. (2007), Sestito et al. (2008). Figure 1 shows 


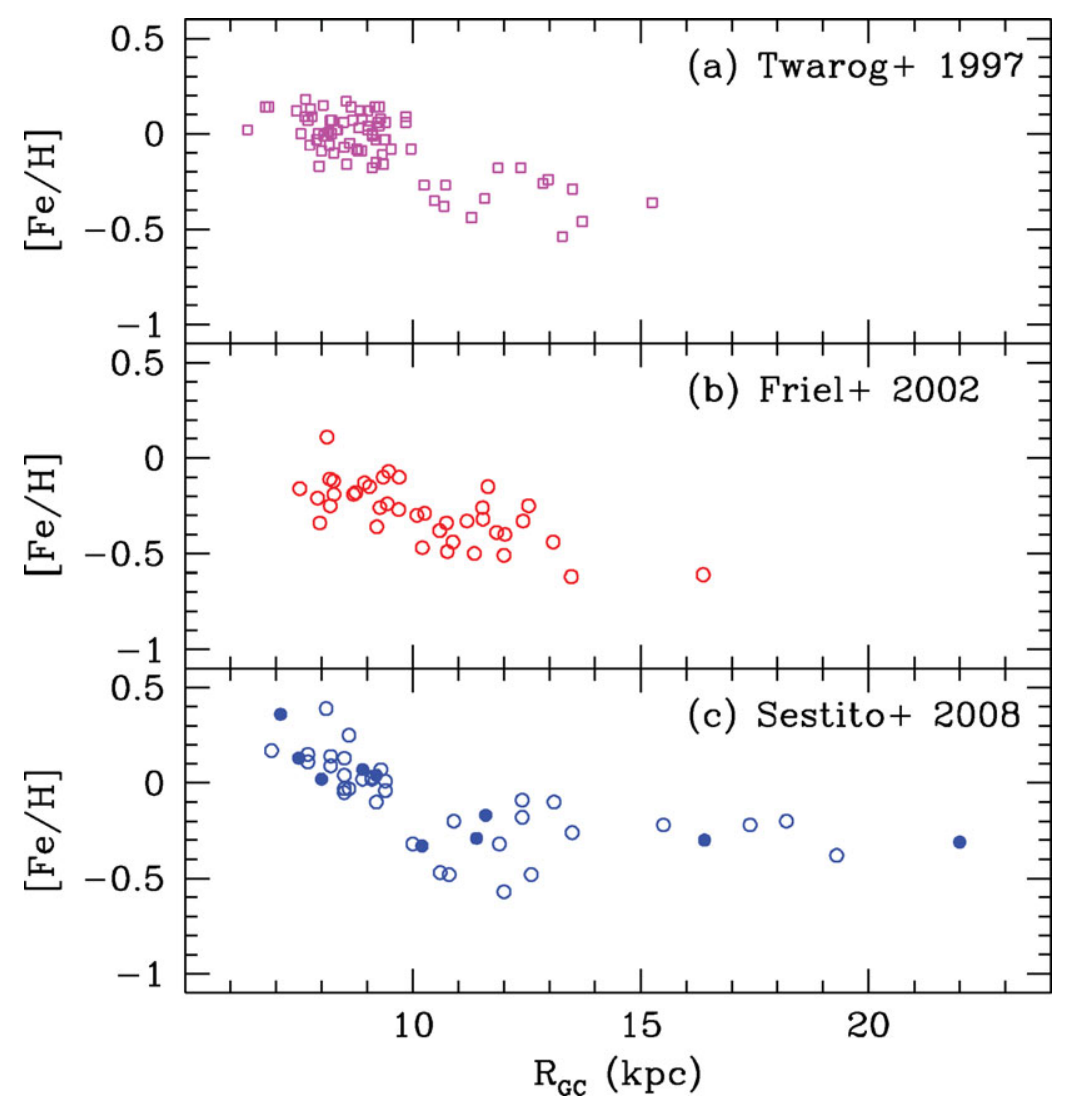

Figure 1. Radial metallicity gradient measured from OCs. (a) Results based (mostly) on DDO photometry from Twarog, Ashman \& Anthony-Twarog (1997), that the authors interpret as a step distribution, with OC's inside $\mathrm{R}_{\mathrm{GC}}=10 \mathrm{kpc}$ with solar $[\mathrm{Fe} / \mathrm{H}]$ and $\mathrm{OCs}$ farther away with $[\mathrm{Fe} / \mathrm{H}] \simeq-0.3$. (b) Results based on low resolution spectroscopy from Friel et al. (2002), where a single radial gradient seems evident. (c) Results based on high resolution spectroscopy, adapted from Sestito et al. (2008). The open circles represent literature values (see the paper for all references), while the filled dots indicate the homogeneous analysis of 10 OCs observed with FLAMES, see Sestito et al. (2006), Sestito, Randich \& Bragaglia (2007), Bragaglia et al. (2008), Sestito et al. (2008) for details. This large (but still largely inhomogeneous) dataset seems to indicate a rather steep gradient for the inner part of the disk, followed by a flattening in the outer part.

these three possibilities; the lower panel, including only results based on high resolution spectroscopy, is adapted from Sestito et al. (2008) and includes more than 40 OCs, one quarter of them with metallicities derived in an homogeneous fashion by the same group.

\section{The BOCCE survey}

Even in the last case described above we are dealing with a rather inhomogeneous sample; systematic effects can be present and maybe blur the picture. To avoid this, we have started a survey of OCs to derive in the most precise and homogeneous way their main parameters: age, distance, reddening, metallicity (and detailed abundances). We 


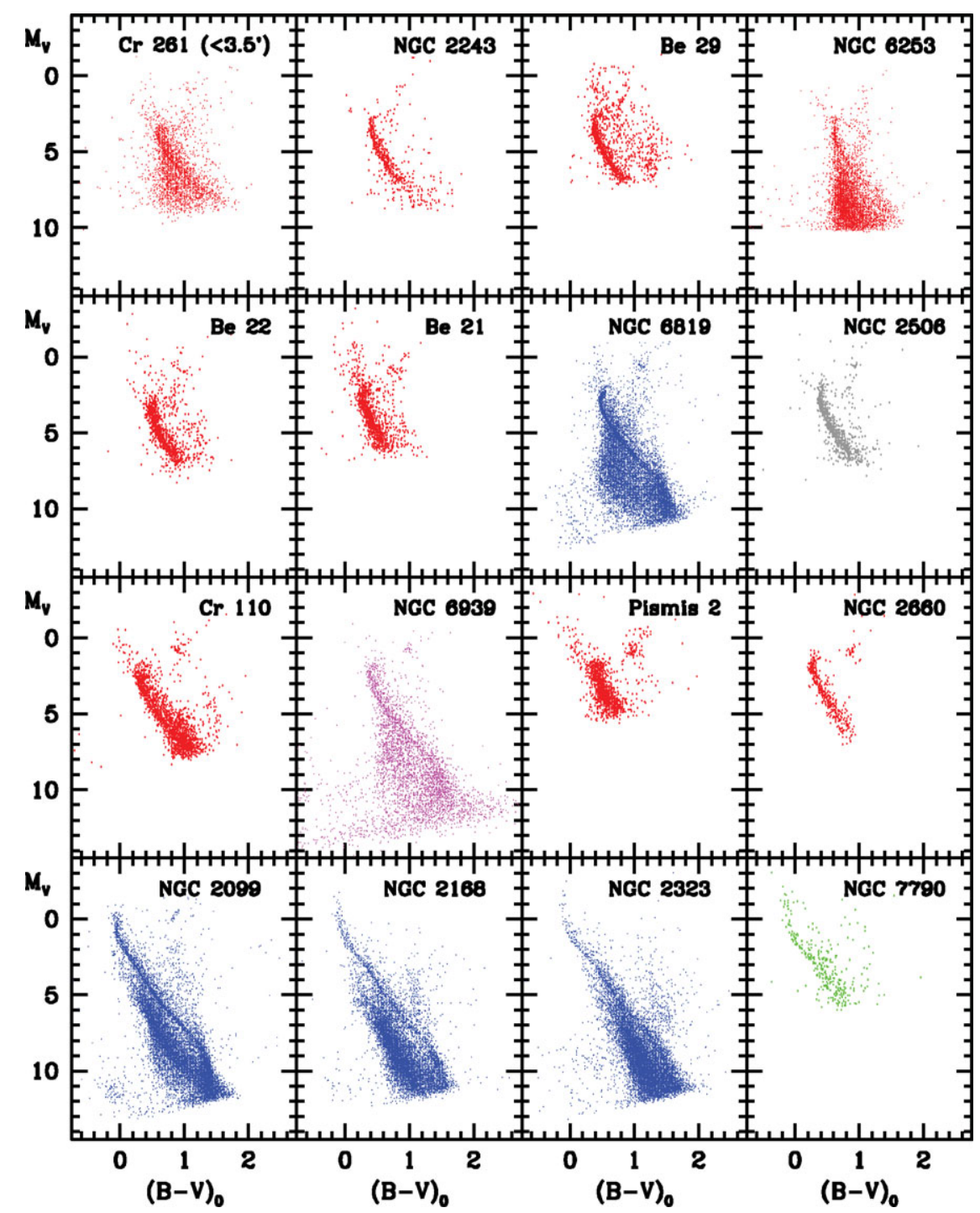

Figure 2. CMDs of the 16 BOCCE OCs presented in Bragaglia \& Tosi (2006), shown in decreasing age order. The different colors indicate the source of data: red for ESO telescopes, magenta for the TNG, blue for the CFHT, grey for mixed, green for Loiano.

named our survey the "Bologna Open Clusters Chemical Evolution" project (BOCCE in short), since one of our main interests is the study of the chemical evolution of the disk. We are building a sample large enough (about 40 OCs) to be representative of the whole cluster population (in age, metallicity and position in the Galaxy). We employ:

- Photometry and the Synthetic Colour-Magnitude diagrams (CMD) technique to derive at the same time age, distance, reddening and a first indication of metallicity; for a review of the method and results see Bragaglia \& Tosi (2006). This is the more advanced part of our survey; the CMDs for the first 20 OCs analyzed are shown in 
Fig. 2. To build the synthetic CMDs we take into account the photometric errors, the completeness factors, the presence of binaries and the contamination due to field stars. To maintain the highest homogeneity we always use the same three sets of evolutionary trackst, without overshooting and with two treatments of it. With our method we are able to quantify the systematics between results based on different stellar models, and we can also get an homogeneous ranking of the OC population by choosing results based on (any) one of the models.

- Medium resolution spectroscopy to derive radial velocities, hence membership, for stars in crucial evolutionary phases, like the main sequence Turn-Off or the Red Giant Branch; for an application, see e.g. D'Orazi et al. (2006). This is a secondary part of our project.

- High resolution spectroscopy to measure chemical abundances, using both equivalent widths and spectrum synthesis; for a presentation of the method, see Bragaglia et al. (2001), Carretta et al. (2004). We obtain spectra of a few stars per cluster, usually on the Red Clump, and possibly already known to be cluster members. We try to maintain also in this case the most homogeneous procedure, using the same line lists, $g f$ 's, model atmospheres, solar reference values, way to measure equivalent widths, synthesis, and kind of stars. A smaller part of the spectroscopic work has been completed (see Tab. 1 in Bragaglia et al. 2009 for an update). However, recent and planned observations have doubled the set of OCs, and we plan to use also archive data and homogenize results obtained within a parallel program (see Randich et al. 2005 and the already mentioned Sestito et al. 2008).

We have observed clusters with ages from 0.1 to $9 \mathrm{Gyr}, \mathrm{R}_{\mathrm{GC}}$ from about 7 to $21 \mathrm{kpc}$, and metallicity from less than half solar to more than double solar. Fig. 3 (left panels) shows more CMDs for clusters recently analyzed or on which we are presently working. Since we want to study the history of the disk we concentrated on old OCs: we have collected data for a fair fraction of the about 120 OCs with age larger than 1 Gyr found in the Dias et al. (2002) catalogue, see Fig. 3 (right panels).

We have a few very interesting objects in our sample, like Be 17 and NGC 6791‡, which are the oldest OCs known, with an age near 9 Gyr. We also analyzed Be 20 and Be 29, with $\mathrm{R}_{\mathrm{GC}}$ of about 16 and $21 \mathrm{kpc}$, or $\mathrm{Cr} 261$ and NGC 6253 , which lie inside the solar circle; they are very important to define the metallicity gradient. Note also that two of these clusters, NGC 6791 and NGC 6253, are the most metal-rich OCs presently known (with $[\mathrm{Fe} / \mathrm{H}] \geqslant+0.4$ dex, Carretta, Bragaglia \& Gratton 2007).

Our effort to build a large, significant sample of open clusters with ages, distances, metallicities and detailed abundances measured with homogeneous methods is well under way and has already produced interesting results. While we have not yet succeeded in having all the clusters properties measured on the same scale (to guarantee that features are not created or lost because of systematic differences between analyses), we

$\dagger$ Since homogeneity is a key requirement of the project, we choose not to use other models or newer versions of our basic set; we are more interested in the ranking than in the absolute values. If really better tracks appear on the market we can switch to them, but in this case we have to uniformly apply them to the whole sample.

$\ddagger$ We are presently working on NGC 6791, using CFHT data, see Kalirai et al. (2007), that we combine with literature information on other photometric bands and on membership (both from radial velocities and proper motions, the latter thanks to private communication kindly provided by K. Cudworth). Given the extreme metallicity of this cluster, we will have to use new tracks, computed on purpose using the same -or very similar- codes as the other ones. 

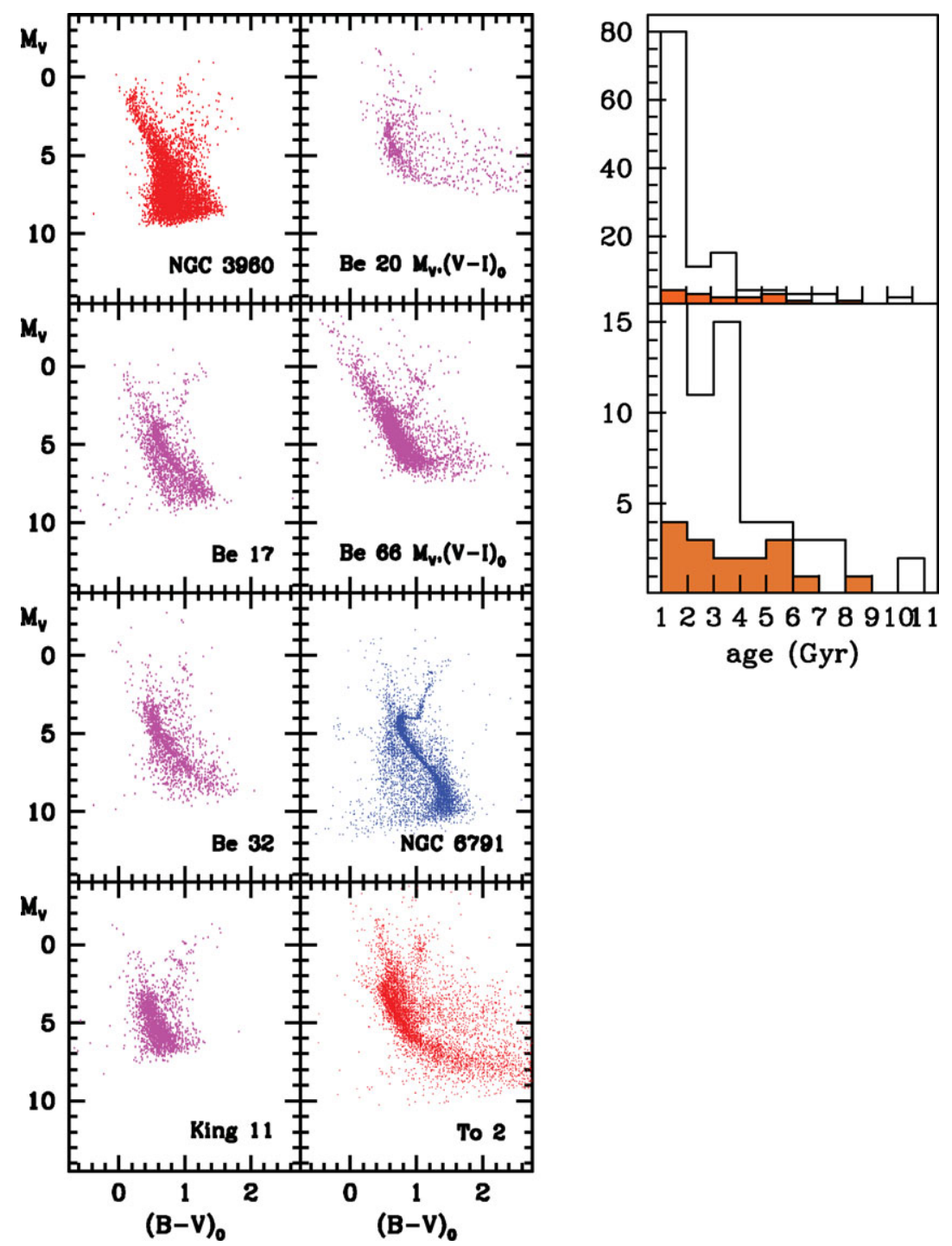

Figure 3. Left: CMD of four more BOCCE OCs published in refereed journals (NGC 3960, Bragaglia et al. 2006a; Be 17, Bragaglia et al 2006b; Be 32 and King 11, Tosi et al. 2007), of two clusters presented at a conference (Be 20, Be 66, Andreuzzi et al. 2008), and of two OCs on which we are presently working (NGC 6791, To 2). Right: Age distribution of the about 120 OCs with ages larger than 1 Gyr in the Dias et al. (2002) catalogue (open histogram) and of the 16 OCs in the BOCCE sample already analyzed and older than 1 Gyr (filled histogram); the lower panel shows an enlargement.

are struggling towards this goal and hope to be able to present a completely homogeneous picture of our sample soon.

The work described is a collaboration of many researchers: Monica Tosi and Eugenio Carretta (INAF - Bologna Observatory), Raffaele Gratton (INAF - Padova Observatory), Gloria Andreuzzi and Luca Di Fabrizio (INAF - Fundación Galilei), Michele Cignoni (Bologna University), Jason Kalirai (STScI), and Gianni Marconi (ESO Chile). 
We thank Paola Sestito and Sofia Randich for useful discussions; we make use here of results obtained in collaboration with them. The WEBDA (created by J.-C. Mermilliod and presently developed by E. Paunzen at http://www.univie.ac.at/webda/) was and will be of invaluable help. Generous allocation of observing time at Italian telescopes (TNG and Loiano), at the CFHT, and at ESO telescopes (La Silla and Paranal) is acknowledged.

\section{References}

Andreuzzi, G., Bragaglia, A., \& Tosi, M. 2008, MemSAI, 79, 657

Bragaglia, A., et al. 2001, AJ, 121, 327

Bragaglia, A. \& Tosi, M. 2006, AJ, 131, 1544

Bragaglia, A., Tosi, M., Carretta, E., Gratton, R. G., Marconi, G., \& Pompei, E. 2006a, MNRAS, 366,1493

Bragaglia, A., Tosi, M., Andreuzzi, G., \& Marconi, G. 2006b, MNRAS, 368, 1971

Bragaglia, A., Sestito, P., Villanova, S., Carretta, E., Randich, S., \& Tosi, M. 2008, A\&A, 480, 79

Bragaglia, A., Carretta, E., Gratton. R., \& Tosi, M. 2009, in: J. Andersen, J. Bland-Hawthorn \& B. Nordström (eds.), The Galaxy Disk in Cosmological Context, in press

Carraro, G., Geisler, D., Villanova, S., Frinchaboy, P. M., \& Majewski, S. R. 2007, A $₫ A, 476$, 217

Carretta, E., Bragaglia, A., Gratton, R. G., \& Tosi, M. 2004, A\&A, 422, 951

Carretta, E., Bragaglia, A., \& Gratton, R. G. 2007, A\& A, 473, 129

D’Orazi, V., Bragaglia, A., Tosi, M., Di Fabrizio, L., \& Held, E. V. 2006, MNRAS, 368, 471

Dias, W. S., Alessi, B. S., Moitinho, A., \& Lépine, J. R. D. 2002, A\& $A$, 389, 871

Friel, E. D. 1995, ARAA, 33, 381

Friel, E. D., Janes, K. A., Tavarez, M., Scott, J., Katsanis, R., Lotz, J., Hong, L., \& Miller, N. 2002, AJ, 124, 2693

Kalirai, J. S., Bergeron, P., Hansen, B. M. S., Kelson, D. D., Reitzel, D. B., Rich, R. M., \& Richer, H. B. 2007, ApJ, 671, 748

Maciel, W. J., Costa, R. D. D., \& Uchida, M. M. M. 2003, A\&A, 397, 667

Randich, S., et al. 2005, ESO Messenger, 121, 18

Sestito, P., Bragaglia, A., Randich, S., Carretta, E., Prisinzano, L., \& Tosi, M. 2006, A\&A, 458, 121

Sestito, P., Randich, S., Bragaglia, A. 2007, A\& $\mathcal{G}$, 465, 185

Sestito, P., Bragaglia, A., Randich, S., Pallavicini, R., Andrievski, S. M., \& Korotin, S. A. 2008, $A \mathscr{E} A, 488,943$

Stanghellini, L., Guerrero, M. A., Cunha, C., Manchado, A., \& Villaver, E. 2006, ApJ, 651, 898 Tosi, M., Bragaglia, A., \& Cignoni, M. 2007, MNRAS, 378, 730

Twarog, B. A., Ashman, K. M., \& Anthony-Twarog, B. J. 1997, AJ, 114, 2556

Yong, D., Carney, B. W., \& Teixera de Almeida, M. L. 2005, AJ, 130, 597

\section{Discussion}

B. WEAVER: Your metallicity errors seem very small. How do you include uncertainties in atomic constants and atmosphere models?

A. BRAgAglia: The internal errors are small. These uncertainties make the systematic part. But as long as we compare clusters analyzed in the same way, the internal errors are the most important. This is why we try to obtain all parameters for the whole sample homogeneously. 
B. NorDSTRÖM: The metallicity gradient that goes up towards the Galactic center seems to be flat from about $10 \mathrm{kpc}$ out. Could you please comment on that? Will yo have more data to see if this is a real effect?

A. Bragaglia: The flattening is seen in other works on open clusters, like those of Yong et al. 2005 (AJ, 130, 597), or Carraro et al. 2007 (A\&A, 476, 217). The exact position of the flattening is still uncertain, at about 10-12 kpc. We are obtaining new data near that $R_{\mathrm{GC}}$ to better define it. 


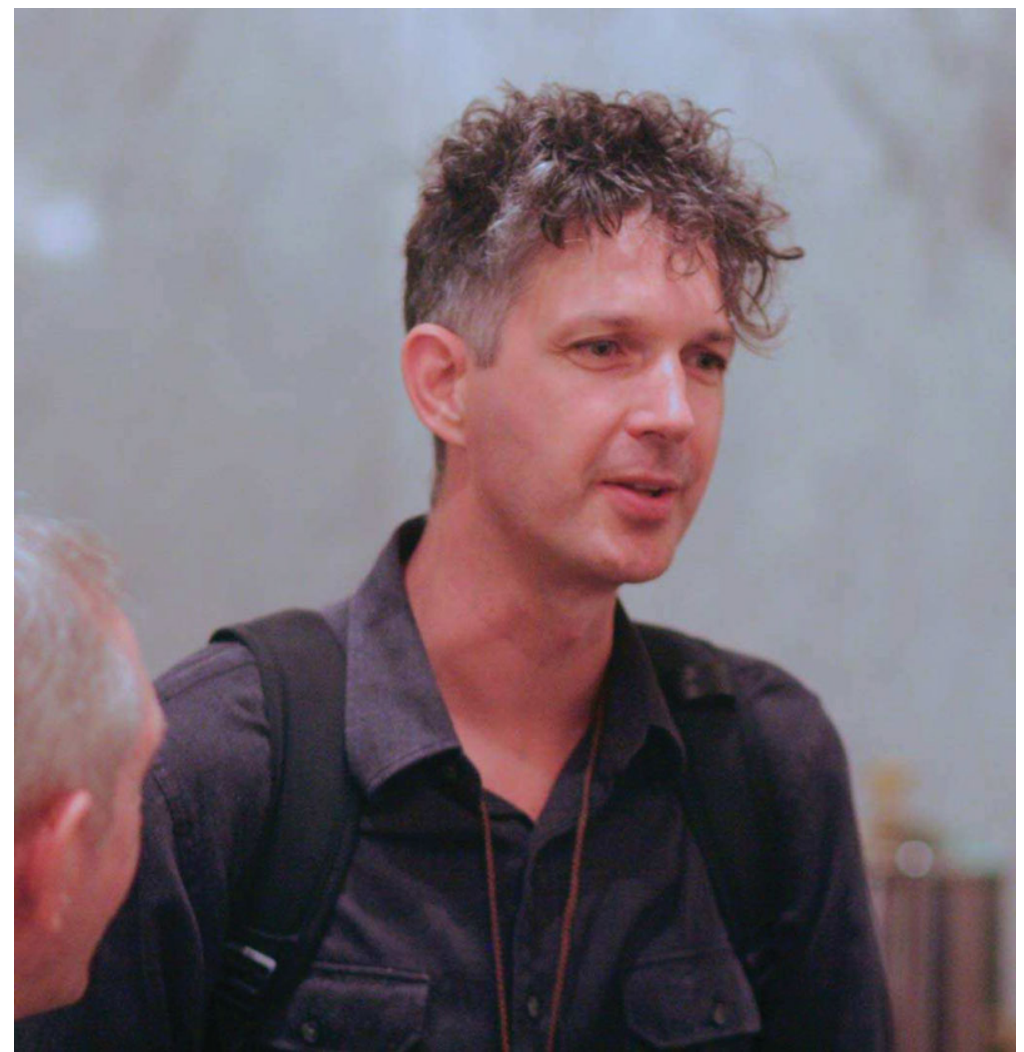

Roelef de Jong

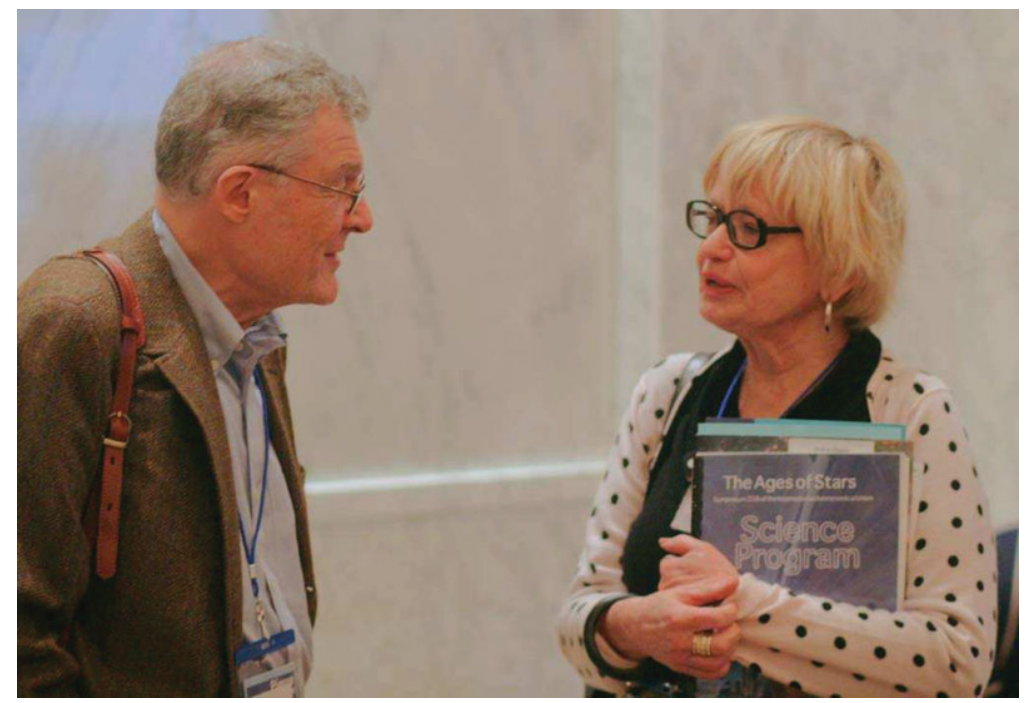

Pierre Demarque and Andrea Dupree 